\title{
Prospectiva de futuro del proceso de enseñanza-aprendizaje del Lenguaje Musical en España
}

\author{
Prospective of the Future of the Teaching-Learning Process of Musical \\ Language in Spain
}

\author{
María José Sánchez Parra \\ Universidad de Castilla-La Mancha \\ mjose.sanchez@uclm.es \\ ORCID iD: https://orcid.org/0000-0003-2161-2746
}

\section{RESUMEN}

Este artículo tiene por objetivo conocer cuál ha sido la evolución del proceso de enseñanzaaprendizaje del Lenguaje Musical, así como una prospectiva de su futuro como materia fundamental en los Conservatorios Profesionales de Música de España. La metodología de investigación aplicada ha sido el análisis de contenido, la entrevista semi-estructurada a expertos de prestigio en la docencia del Lenguaje Musical, los grupos de discusión y la encuesta. Los resultados obtenidos muestran la necesidad de un diseño educativo innovador para la asignatura de Lenguaje musical en los Conservatorios Profesionales de Música de España.

Palabras clave: Lenguaje Musical, $\mathrm{TIC}^{1}$ y música, proceso educativo, conservatorios de música.

\section{AbStract}

The aim of this article is to study the evolution of the teaching-learning process of Musical Language, as well as a prospective of its future as a fundamental subject in the Professional Conservatories of Music of Spain. The applied methodology of this research has been analysis of content, semi-structured interviews to prestigious experts in the teaching of Musical Language, discussion groups and surveys. The results obtained show the need for an innovative educational scheme for the Musical Language subject in the Professional Conservatories of Music of Spain.

Key words: Musical Language, ICT and Music, Educational process, Music Conservatories.

1 Tecnologías de la Información y la Comunicación. 


\section{MARÍA JosÉ SÁNCHEZ PARRA}

Sánchez Parra, M. J. (2018). Prospectiva de futuro del proceso de enseñanza-aprendizaje del Lenguaje Musical en España. Cuadernos de Investigación Musical, 5, 137-160.

\section{INTRODUCCIÓN}

El tema de este artículo es mostrar la investigación llevada a cabo sobre el proceso de enseñanza-aprendizaje del Lenguaje Musical en los Conservatorios Profesionales de Música de España ${ }^{2}$, teniendo en cuenta el pasado, el presente y el futuro de dicha asignatura.

\section{JUSTIFICACIÓN}

Entre los estudiantes de música de los conservatorios profesionales ha existido un cierto rechazo a la materia del Solfeo, actualmente conocido como Lenguaje Musical, que ha impedido una mayor profundidad en su conocimiento. Valencia Déniz y Ventura del Rosario (2003), dicen que la asignatura de Lenguaje Musical ha despertado cierta apatía hacia su estudio por parte de los estudiantes, llegando incluso al abandono de los estudios musicales. Ante esta situación preocupante nos hizo preguntarnos si chabía cambiado el proceso de enseñanza-aprendizaje del Lenguaje Musical y en qué aspectos desde el Decreto de $1942^{3}$ hasta la actualidad? Este punto de partida en la investigación nos hizo reflexionar sobre el objeto de estudio y como consecuencia surgieron nuevas preguntas referentes a: la metodología y los recursos didácticos utilizados, la formación de los profesores, las dimensiones que engloban el Lenguaje Musical, la organización y la coordinación entre el profesorado y a la valoración social de la formación musical en España.

\section{MARCo TEÓRICO}

Para poder entender el Lenguaje musical, como objeto de estudio, debemos profundizar en cada uno de sus elementos. En este apartado

\subsection{EDUCACIÓN AUDITIVA}

La percepción auditiva es uno de los pilares fundamentales en el aula de Lenguaje Musical ya que desarrolla la musicalidad y es imprescindible para un proceso de enseñanzaaprendizaje musical completo y significativo. Por ello, es muy importante recibir una buena educación auditiva desde edades muy tempranas y no caer en el error de prorrogar dicha educación del oído a enseñanzas profesionales o superiores, donde los alumnos ya tienen una edad más avanzada.

Pedagógicamente, el trabajo auditivo en el aula requiere de una metodización e insistencia específica ya que, para conseguir una agilidad auditiva cada vez mayor en los

\footnotetext{
${ }^{2}$ En la investigación han participado docentes de Lenguaje Musical de todas las comunidades autónomas, exceptuando Asturias e Islas Baleares. La participación se ha estado estratificada atendiendo al número de conservatorios profesionales existentes en cada una de las Comunidades Autónomas.

${ }^{3}$ Decreto de 15 de junio de 1942 sobre organización de los Conservatorios de Música y Declamación.
} 


\section{ProspeCTIVA DE FUTURO DEL PROCESO DE ENSEÑANZA-APRENDIZAJE DEL LENGUAJE MUSICAL EN ESPAÑA}

alumnos, el profesor encargado de educar el oído tiene que realizar una selección minuciosa de ejercicios que sean trabajados con mucha constancia. $Y$ es que no podemos pasar por alto que la audición es una de las manifestaciones más claras de pensamiento musical.

Algunos pedagogos, como Edgar Willems o Jaques Dalcroze, defienden que el hecho de escuchar música es muy beneficioso para la formación auditiva y que si, además, los sonidos son relacionados con imágenes, nombres, colores o números, resultará más sencillo de reconocer y recordar.

Por otro lado, el entrenamiento auditivo, puede desarrollarse con éxito si se utilizan instrumentos musicales como el instrumental Orff o las campanillas dispuestas en escala que recomienda Edgar Willems.

Este autor, en su libro El oído musical: la percepción auditiva del niño (2001), presenta la audición musical gravitando sobre tres aspectos:

a) La sensorialidad auditiva, en la que oír es inconsciente- subconsciente. Pedagogos de todo el mundo apoyan la idea de que la comprensión de la melodía y armonía tiene que apoyarse en una buena educación sensorial auditiva. Las cualidades del sonido serán descubiertas cuanto mejor desarrollada esté la sensorialidad auditiva. Juan Amos Comenius (citado por Willems 2001) comenta que no había nada que no hubiera pasado antes por los sentidos y que para que un alumno aprendiera a conocer los sonidos debía ejercitarse primero los sentidos. La experiencia sensorial es un punto de partida necesario para despertar otras facultades humanas. Grandes sabios y pedagogos como J. J. Rousseau, Pestalozzi, Decroly, Montessori, etc., han tratado el tema de la importancia del desarrollo de la agudeza sensorial.

b) La sensibilidad auditiva, escuchar es inconsciente-subconsciente, ya que engloba la acción de oír, pero implica también intelectualidad por lo que también es consciente. La audición necesita de atención y concentración, ya que desde el momento que estamos desarrollando la sensorialidad, también estamos trabajando la sensibilidad.

c) La sensibilidad afectivo-auditiva, comienza en el momento en el que pasamos del acto pasivo y objetivo de oír a escuchar, más activo y subjetivo. Si antes decíamos que la sensorialidad es el punto de partida, la sensibilidad auditiva es como el centro, ya que "por la sensibilidad auditivo- afectiva entramos en el campo melódico; gracias a ella el hombre puede cantar su alegría, sus dolores, sus esperanzas o más simplemente, su amor por la belleza sonora." (Willems, 2001: 55).

Por otro lado, la Inteligencia auditiva, se basa en entender lo que se oye, aspecto que necesita tener una sensorialidad y sensibilidad bien desarrolladas. Tenemos que ser capaces de relacionar lo que se ha captado con vivencias anteriores e interpretar lo que se ha escuchado. Para poder hacer esto, es necesario el conocimiento técnico e intelectual del lenguaje a emplear. Consiste en relacionar la conexión entre significantes y significados.

Una de las herramientas más utilizadas en el aula de Lenguaje Musical para el desarrollo auditivo es el dictado, el cual Elena Berrón (2016) define como un proceso en el que los alumnos escuchan una serie de sonidos medidos y que tienen que anotar en un 


\section{MARÍA JosÉ SÁNCHEZ PARRA}

papel, aunque también pueden dar una respuesta oral o interpretativa en vez de escrita. Para Clara Misas y Alejandro Tobón (2007), el objetivo del dictado es comprender, conectar y asociar, y es que tenemos que entender el dictado como un proceso que evoluciona constantemente y que por tanto debe ser revisado permanentemente.

Terminamos este apartado con la idea que tiene sobre el dictado Encarnación López de Arenosa (2004), que advierte que la actividad del dictado no consiste en adivinar lo que se oye y que el dictado no es nada si no sirve para un entendimiento del Lenguaje Musical en todos sus aspectos.

\subsection{EDUCACIÓN VOCAL}

Respecto a la Educación Vocal, Willems en su obra Las bases psicológicas de la educación musical dice que "el canto desempeña el papel más importante en la formación musical de los principiantes, ya que es el mejor medio para desarrollar el oído interno, clave de toda verdadera musicalidad" (2001: 33).

Begoña Torres (2013), ha investigado sobre los aspectos beneficiosos de la música y dice que gracias a la educación vocal se desarrollan las cualidades vocales, se descubren las posibilidades de la voz y con ello se aprenden hábitos de educación vocal como puede ser una buena respiración y emisión de la voz.

Cantar es una conducta muy concreta del ser humano, es una de las formas más antiguas de expresión. Además, hay que hacer incidir en la idea de que cualquier persona puede aprender a cantar, es una actividad que engrandece al pueblo que lo practica y ayuda a construir y a desarrollar una importante base cultural (Barceló, 1995). Tenemos la capacidad de expresarnos y comunicarnos con los demás a través de la voz. Los niños son musicales por naturaleza y por tanto toman la voz como uno de los instrumentos principales para poder comunicarse con el entorno que les rodea (Shehan Campbell, 1998).

Dentro de la enseñanza del Lenguaje Musical, la voz es uno de los órganos más importantes, ya que debe utilizarse como elemento de aprendizaje de emisión y reconocimiento auditivo del sonido (Perandones, 2010).

Si pretendemos dar a nuestros alumnos una formación completa, debemos tener muy en cuenta la canción como fuente de riqueza cultural. Muchos de los grandes pedagogos de la Escuela Nueva, coinciden en afirmar los grandes beneficios que se obtienen gracias a la práctica del canto.

Es manifiesto que la mejor manera de aprender música consiste en hacer música, y nuestra propia vOz es el instrumento más completo que tenemos para hacer música y disfrutar con ella. Los profesores, gracias al canto, podemos introducir a los alumnos en el conocimiento práctico de elementos del Lenguaje Musical para después analizarlos teóricamente desde los ejemplos ya experimentados.

Parece innegable que, si el canto es una actividad recomendable en la clase de música del sistema educativo general se haga más evidente, con mayor motivo, en un aula de música de las enseñanzas especiales.

El profesor encargado de la educación vocal debe confeccionar un repertorio de 


\section{Prospectiva de FUtURo Del PROCESO DE ENSEÑANZA-APRENDIZAJE DEL LENGUAJE MUSICAL EN ESPAÑA}

canciones didácticas para desarrollar la enseñanza de la asignatura a través del canto. Pero no solo debe enseñar a cantar, sino que también debe enseñar a entonar. De esta forma, el estudiante se obliga a ser consciente en el canto con justeza melódica provocando que el pensamiento musical resulte imprescindible, diferenciándose del simple hecho de cantar que es algo más genérico y que puede ser consciente o no.

\subsection{EDUCACIÓN RÍTMICA}

La música es un arte auditivo cuya naturaleza es dinámica, por lo tanto, necesita ponerse en movimiento a través del tiempo para poder ser captada y percibida. De este modo, el tiempo es un factor inseparable del discurso musical el cual necesitará de una primera ordenación: el ritmo. Gracias a él, los demás elementos como la melodía, la armonía y el timbre, cobran vida y sentido.

El ritmo como primer elemento de la música, es fácilmente integrable en la educación musical por su carácter fisiológico, por eso casi todas las pedagogías del siglo XX hacen referencia al ritmo de una manera u otra.

Para Dalcroze, la rítmica es la base de su método. Pretende que los alumnos vivencien el Lenguaje Musical a partir de movimientos rítmicos. En el método de Orff el estudio del ritmo parte de la voz. Uno de los métodos empleados por Kodaly para el estudio del ritmo es la improvisación rítmica sobre elementos conocidos.

El profesor encargado de la educación rítmica en el aula de Lenguaje musical, además de practicar la lectura compaseada, deberá trabajar la percepción, la imitación y la improvisación.

En su estudio sobre la percepción musical, Nicole Du Saussois, Marie-Bernadette Dutilleul y Helene Gilabert (1992) apuntan que, la percepción-musical no es un fenómeno pasivo que pone en funcionamiento la receptividad de un aparato sensitivo ante las estimulaciones que llegan del exterior. Es una actividad psicológica, como ya demostró Jean Piaget. La percepción musical participa del aspecto cognitivo y del emocional.

En cuanto a la imitación, Meyer Howard Abrams (1971) señala que es un término relacional, ya que implica la existencia de dos objetos, el modelo y su representación, vinculados entre sí. En el caso del aula de música, la imitación no sería otra cosa que la repetición por parte de los alumnos de cada uno de los ejemplos realizados por el profesor.

Por último, la improvisación no es un complemento más o menos necesario en la formación de un músico, sino que es la esencia misma de su educación musical y es que improvisar es hablar musicalmente controlando el lenguaje, tal y como explica Emilio Molina (1998). La improvisación proporciona acceso directo al proceso creativo (Díaz y Riaño, 2007).

\subsection{EDUCACIÓN TEÓRICA}

Desde la antigüedad, existen numerosos tratados sobre la Teoría Musical, pues ésta ha sido considerada durante siglos como la base de la educación musical. Afortunadamente, se conservan un conjunto admirable de libros que abordan cuestiones teóricas de lo más interesantes. Gracias a los autores de estos tratados, hemos podido seguir el desarrollo de la 


\section{MARÍA JosÉ SÁNCHEZ PARRA}

teoría musical paso a paso. Hoy podemos saber que los pitagóricos consiguieron resultados muy positivos en cuanto a sus estudios de acústica, formulando principios que la física moderna ha confirmado. Por otro lado, el Tratado de Música de Boecio (2005), que consta de cinco libros, supone la fuente más importante y amplia para la transición del pitagorismo. Casiodoro (1895), quien se dedicó a investigar y a enseñar a sus monjes del Monasterio Vivarium, escribió un tratado en el que puede verse como el pitagorismo ya está superado, pero al no tratarse de un tratado demasiado extenso, los datos no son muy abundantes. Principalmente hace una clasificación de los instrumentos, aborda el tema de las consonancias y del sistema tonal. Más adelante, San Isidoro, considerado un hombre virtuoso, consiguió reunir una biblioteca de numerosos volúmenes llenos de sabiduría, fruto de sus investigaciones, y donde podemos encontrar información sobre la división de la música, definiciones de diafonía y sinfonía, clasificación y características de las voces e instrumentos, y el origen y ejecución del canto litúrgico.

Por lo tanto, debemos a Boecio, Casiodoro y San Isidoro (2004) la difusión de las doctrinas clásicas. A partir de ellos, surgieron otros tratadistas entre los que cabe destacar a Guido De Arezzo, considerado una de las figuras claves en la evolución de la Teoría Musical. Posee dos obras fundamentales, el Micrologus (1991) que es su tratado más completo, donde habla del estudio de los sonidos, la división del monocordio, intervalos, modos, composición de melodías, notación, diafonía, etc. Mientras que el tratado Epistola Michaeli monacho de ignotu cantu (2015) expone su ya famosa solmisación. Guido utilizaba el himno dedicado a San Juan ya que era muy conocido entre los cantantes de la época y porque las primeras sílabas de cada uno de los seis versos que lo formaban coincidían con la escala diatónica.

Pero en estos tratados no se dan nociones didácticas sobre cómo enseñar estos conceptos teóricos, ya que se dedican únicamente a explicar de forma puramente teórica, conceptos como la notación, los intervalos, la forma musical, etc.

\subsection{El uso de LAS TIC COMO RECURSO PARA EL LENGUAJE MUSICAL}

Quienes no sepan desenvolverse en la cultura y tecnología digital de un modo inteligente (saber conectarse y navegar por redes, buscar información útil, analizarla y reconstruirla, comunicarla a otros ciudadanos) no podrán acceder a la cultura y a los entornos laborales de la Sociedad de la Información, por lo que tendrán una alta probabilidad de quedar marginados en la sociedad del siglo XXI, afirman Felipe Gértrudix y Manuel Gértrudix (2007). Nuestra sociedad está cambiando, y esto repercute en cómo conocemos, en cómo aprendemos y en los espacios en los cuales llegamos a aprender (Cabero, 2007).

Ignacio Sustaeta y Ma Pilar Domínguez-Alcahud, (2004) opinan que la introducción en la sociedad actual de estas nuevas tecnologías está suscitando cambios en las generaciones de hoy, hasta tal punto que los alumnos que se encuentran en edad escolar, no pueden imaginar cómo era la vida cuando no existían estos medios.

Los autores anteriormente citados, no iban desencaminados en sus estudios, pues más o menos diez años después de sus trabajos, podemos hablar del vertiginoso crecimiento que han tenido las TIC en todos los ámbitos de la vida y en especial en el 


\section{Prospectiva de FUtURo Del PROCESO DE ENSEÑANZA-APRENDIZAJE DEL LENGUAJE MUSICAL EN ESPAÑA}

campo de la educación.

El desarrollo acelerado de la Sociedad de la Información está dando lugar a retos para la enseñanza-aprendizaje que eran impensables hace unos años (Marchesi, 2012). Quizás lo más destacable sea que las nuevas generaciones de estudiantes no han tenido que acceder a las nuevas tecnologías porque han nacido con ellas y se enfrentan al conocimiento de maneras distintas a las del pasado. Esto supone un gran desafío para los profesores, para las escuelas, para los responsables educativos y para los gestores de las políticas públicas.

Poco a poco, las Tecnologías de la Información y Comunicación (TIC), se han ido integrando en los centros educativos. Al principio, los profesionales de la educación reflexionaban sobre lo adecuadas o no que resultaban las tecnologías para el aprendizaje, pasados los años, analizan el uso de estas tecnologías y su vinculación a las teorías de aprendizaje, junto a propuestas metodológicas para su implementación. El uso de las TIC no necesariamente implica la implementación de una determinada metodología de enseñanza- aprendizaje. En numerosas ocasiones se producen procesos educativos que integran las TIC siguiendo una metodología tradicional, en la que el alumno recibe la información que transmite el profesor y en la que se valora por encima de cualquier cosa la capacidad de atención y la memoria de los alumnos. Sin embargo, tienen en las TIC un fuerte aliado aquellos profesores que desean guiar los aprendizajes de los alumnos, fomentando la interacción y el aprendizaje colaborativo siguiendo los postulados del constructivismo social de Vygostsky o el aprendizaje por descubrimiento de Bruner.

El impacto que están teniendo las TIC sobre la educación da lugar a uno de los mayores cambios en el ámbito de la Educación. En el aula se abre una nueva ventana a través de Internet, que nos permite el acceso a múltiples recursos, informaciones y la posibilidad de comunicarnos con otros $y$, por tanto, la posibilidad de conocer personalidades de opiniones diversas.

En la actualidad contamos con un gran número de aplicaciones musicales que nos pueden ayudar en el Lenguaje Musical en distintos aspectos como son la edición musical, el entrenamiento auditivo, el acompañamiento musical, etc. $\mathrm{Y}$ es que Internet ha supuesto una revolución en cuanto a la facilidad para compartir el conocimiento, por ejemplo, podemos consultar blogs de otros profesores, ver videos tutoriales en Youtube y hasta la Realidad Virtual puede ser aplicada a la formación musical tal y como expone en sus investigaciones Luis Ponce de León y Pilar Lago (2015).

La utilización de las TIC en la clase de Lenguaje Musical, tiene por objetivos fomentar el interés, optimizar el tiempo de la clase y guiar el estudio en casa por parte de los alumnos. Actualmente, existen alternativas como complemento al proceso de enseñanza-aprendizaje del Lenguaje Musical más tradicional. A continuación, hacemos un resumen de algunas de las investigaciones que se han llevado a cabo en el campo del Lenguaje Musical.

Ana Laucirica, José Antonio Ordoñana y Nerea Muruamendiaraz (2009), ya se dieron cuenta que las nuevas tecnologías estaban ocupando un importante y creciente espacio en 


\section{MARÍA José SÁNCHEz PARRA}

las últimas décadas. Desarrollaron una investigación sobre los procesos de aprendizaje rítmico en el periodo que comprende las Enseñanzas Elementales de Música en Conservatorios y la contribución que las nuevas tecnologías podían ejercer sobre los mismos. Las conclusiones a las que llegaron fueron:

1. Las nuevas tecnologías permiten acceder a la adquisición del conocimiento desde diferentes perspectivas (visual, auditiva, espacial...), lo que ayuda a individualizar el aprendizaje en lo que se refiere a las inteligencias múltiples. De esta manera, las diferentes tendencias cognitivas son atendidas con prioridad en alguna de las actividades que se lleguen a realizar.

2. En el mundo actual los medios informáticos forman parte de la vida cotidiana de niños y niñas, tanto en su faceta académica como lúdica. Su uso en los estudios musicales revertirá en una mayor atracción hacia los mismos y en una mejora en el rendimiento individual y colectivo.

3. Desde el punto de vista cognitivo, entre las edades de ocho y doce años, el ser humano se encuentra en un momento idóneo para relacionarse con el exterior a través de sistemas de representación formal, lo que facilita el uso de programas informáticos con estrategias de aprendizaje basadas en imitación, reconocimiento y lectura de esquemas construidos a través del código musical occidental (p. 58).

Mónica Balo, Pilar Lago y Luis Ponce de León (2014), tomaron como aliadas a las TIC para mejorar las experiencias ante el dictado musical con alumnos de los Conservatorios Profesionales de Música de Madrid, considerando que la aplicación de las TIC en el trabajo de los dictados musicales puede ser una de las estrategias para favorecer la motivación de los alumnos y conseguir que éstos vivan con mayor ilusión su proceso de aprendizaje.

María del Mar Bernabé (2015), llevó a cabo una metodología de sesiones musicales digitales, desarrolladas en Conservatorios Profesionales de Música, centrada en la utilización de la Pizarra Digital. Lo que pretendían era favorecer una metodología más participativa captando el interés de los alumnos hacia la asignatura de Lenguaje Musical, ya que tradicionalmente ha sido considerada más teórica. El estudio demostró que la pizarra digital ofrece interesantes posibilidades para el proceso de enseñanza/aprendizaje en los Conservatorios Profesionales de Música.

Hoy podemos afirmar que cada vez son más los profesores que emplean las TIC en el aula de Lenguaje Musical, pero este hecho, no implica que la utilización de los sistemas tradicionales deje de ser válidos. Tal y como afirman en su investigación Juan Rafael Hernández Bravo, José Antonio Hernández Bravo y Miguel Ángel Milán Arellano (2015), si hay ciertas actividades que se desarrollan de forma tradicional de mejor o de igual manera que con las nuevas, no hay que descartarlas.

En resumen, podemos decir que los alumnos se sienten más motivados al utilizar las TIC en el aula de Lenguaje Musical. Además, compartir recursos en Internet, supone acercar el aula a las familias y fomentar la colaboración e intercambio de información entre profesores de la materia.

\section{Metodología}




\section{ProspeCtiva de FUtURo DEL PROCESo de ENSEÑANZA-APRENDIZAJE DEL LENGUAJE MUSICAL EN ESPAÑA}

Para esta investigación utilizamos un modelo de metodología mixta, cualitativacuantitativa y las técnicas e instrumentos de recogida de información fueron cuatro entrevistas en profundidad, a cuatro de los catedráticos de Lenguaje Musical de este país. Con los datos obtenidos se establecieron las dimensiones de investigación y con ellas la elaboración de dos focus group, el primero tuvo lugar en el Conservatorio Profesional de Música Arturo Soria de la Comunidad de Madrid en el que participaron cinco profesores de Lenguaje Musical actualmente en activo, y el segundo se llevó a cabo en la Universidad de Castilla-La Mancha en la Facultad de Educación de Toledo, en el que participaron cinco profesores de Lenguaje Musical actualmente en activo. Como resultado de estos dos focus group se elaboró un cuestionario online que fue enviado a todos los profesores de Lenguaje Musical de los Conservatorios Profesionales de Música de España, lo que nos proporcionó unos datos masivos sobre el pasado, el presente y el futuro de la asignatura de Lenguaje Musical. De los 131 Conservatorios Profesionales oficiales de música en España (MECD, 2016), la población es de 337 profesores de Lenguaje Musical y la muestra alcanzada ha sido de 155.

Esta triangulación nos ha proporcionado un análisis más exhaustivo del trabajo de investigación. Las diferentes técnicas e instrumentos de recogida de información fueron naciendo a partir de los resultados anteriores.

A continuación, se muestra la encuesta elaborada a partir de los datos obtenidos en los focus group y que, como ya hemos indicado fue enviada a todos los Conservatorios Profesionales de Música de España.

Estudio y Análisis del Proceso de Enseñanza-Aprendizaje del Lenguaje Musical en los Conservatorios
\[ \text { Profesionales de Música } \]
Bienvenido/a:
El Grupo de Investigación Ciberimaginario de la Facultad de Educación correspondiente a la Escuela Internacional de Doctorado de
la Universidad de Castilla-La Mancha está llevando a cabo una investigación sobre el "Estudio y Análisis del Proceso de Enseñanza-
Aprendizaje del Lenguaje Musical en los Conservatorios Profesionales de Música".
Dentro de dicha investigación, se ha diseñado un cuestionario para profesores en activo que están ahora mismo trabajando en el lenguaje
musical. El cuestionario es complentamente anónimo y está compuesto por varios bloques, dentro de los cuales encontrarás las diferentes
preguntas a responder.
Asi pues, solicitamos tu colaboración mediante la cumplimentación del siguente cuestionario.
Muchas gracias.
Siguiente »»


MARÍA JOSÉ SÁNCHEZ PARRA

Estudio y Análisis del Proceso de Enseñanza-Aprendizaje del Lenguaje Musical en los Conservatorios Profesionales de Musica
$0 \%$ $100 \%$

\section{DATOS SOCTODEMOGRÁFICOS}

Lugar de residencia (Comunidad Autónoma y Provincia):

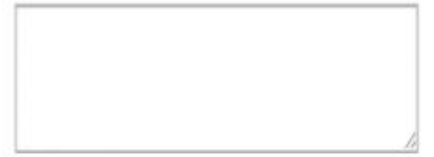

Edad:

Soilo se aceotan nimeros en este campo

Género:

Pemenino Masculino

«Previo Siguiente »

\section{LA ENSEÑANZA RECIBIDA}

Señale las dimensiones de la asignatura de Lenguaje Musical que se impartían cuando usted realizó sus estudios de Música:

Marque las entradas que correspondan
Audición
Teoria
Ritmo
Entonación

¿Cómo se impartían dichas dimensiones?

Seleccione una de las siguientes opciones

Cada una por separado

Combinadamente

Del 1 al 10 (siendo 1 el valor más bajo y 10 el más alto)

¿Qué importancia diría usted que se daba a cada una de ellas?

$\begin{array}{llllllllll}1 & 2 & 3 & 4 & 5 & 6 & 7 & 8 & 9 & 10\end{array}$

Audición 010101010101010100

Teoría 0.000000000

Ritmo 0101001010010010

Entonación 00000000000

¿Se enseñaba metodología didáctica?

Si No 
ProsPeCTIVA DE FUTURO DEL PROCESO DE ENSEÑANZA-APRENDIZAJE DEL LENGUAJE MUSICAL EN ESPAÑA

\begin{tabular}{l} 
La enseñanza de la teoría y la práctica se realizaba: \\
Seleccione una de las siguientes opciones \\
Primero se daba teoría y luego se pasaba a la práctica \\
Primero se daba práctica y luego se pasaba a la teoría \\
Unas veces la teoría precedía a la práctica y viceversa \\
Respecto a la evolución de los planes de estudio, usted cree que: \\
Seleccione una de las siguientes opciones \\
Han cambiado sustancialmente \\
Ho han camberimentado algunas modificaciones \\
En caso de que sí se hayan producido cambios, icómo los calificaría usted? \\
Seleccione una de las siguientes opciones \\
En general los cambios han sido para mejor \\
En general los cambios han sido para peor \\
En algunos casos han mejorado y en otras han empeorado \\
En algunos casos los cambios han supuesto mejoras \\
¿Cuáles han sido esos cambios? (Respuesta abierta): \\
\hline
\end{tabular}

LA IMPARTICIÓN DE LA ASIGNATURA EN LA ACTUALIDAD

Respecto al método de enseñanza de Lenguaje Musical, crees que:

Seleccione una de las siguientes opciones

Ha cambiado sustancialmente

Ha experimentado algunas modificaciones

No ha cambiado prácticamente nada

En caso de que sí se hayan producido cambios, ¿cómo los calificarias?

Seleccione una de las siguientes opciones

En general los cambios han sido para mejor

En general los cambios han sido para peor

En algunos casos los cambios han supuesto mejoras

En algunos casos los cambios han supuesto desmejoras

Respecto al alumnado, consideras que:

Seleccione una de las siguientes opcione

Ha cambiado sustancialmente

- Ha experimentado algunos cambios

No ha cambiado prácticamente nada

En caso de que sí se hayan producido cambios, ¿cómo los calificarías?

Seleccione una de las siguientes opcione

En general los cambios han sido para mejor

En general los cambios han sido para peor

En algunos casos los cambios han supuesto mejoras

En algunos casos los cambios han supuesto desmejoras

¿Cuáles han sido dichos cambios? (Respuesta abieta):

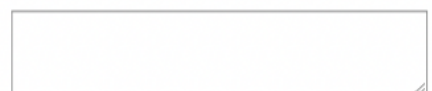

El nivel musical del alumnado es:
Seleccione una de las siguientes opciones

Mejor que el de antes

Igual que el de antes

Mayor que el de antes 


\section{MARÍA JoSÉ SÁNCHEZ PARRA}

\section{LA IMPARTICIÓN DE LA ASIGNATURA EN EL FUTURO}

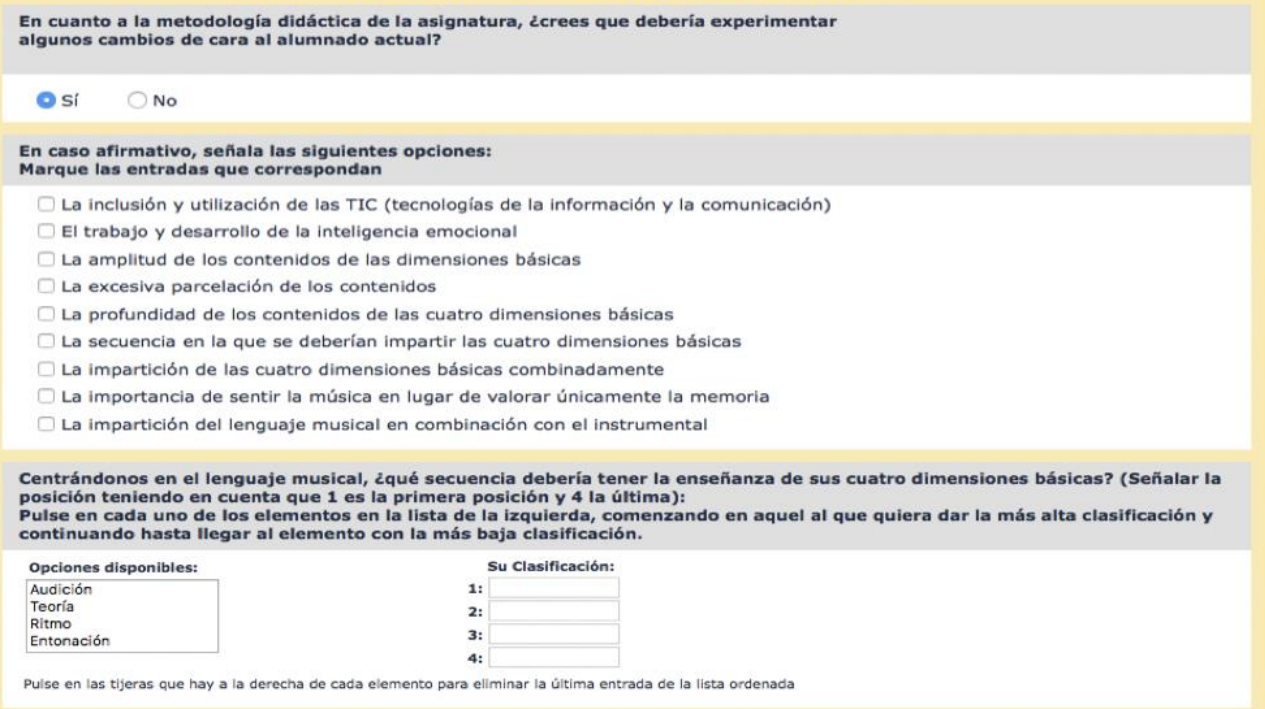

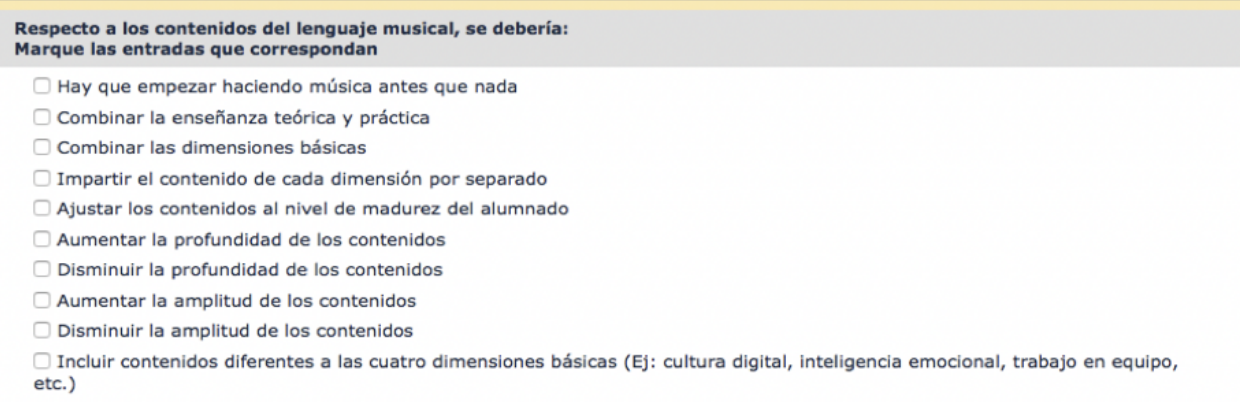

Considerando las dimensiones mencionadas de manera separada, zcuál es el grado de importancia que consideras que tiene cada una? (Siendo 1 la importancia menor y 5 la importancia mayor)

Audición
Crees que la importancia de estas dimensiones debería estar en función de la especialidad del alumnado?
Sí No
Cn materia de coordinación de la asignatura, zcuáles crees que son las opciones más convenientes?
Marque las entradas que correspondan
Una programación de contenidos mínimos a nivel de centro de la asignatura
Una amplia programación a nivel de centro que incluya la mayoría de los aspectos que componen la asignatura más allá de los
contenidos mínimos
La creación de grupos de trabajo entre profesorado de diferentes asignaturas para la coordinación de los programas de estudio
(en concreto con los profesores de instrumento) y la planificación de los contenidos de las asignaturas
La coordinación entre los profesores de la asignatura para que la asignatura tenga una estructura común
La coordinación entre el profesorado de diferentes asignaturas
Es mejor que cada profesor se organice por su cuenta
El mantenimiento de reuniones con profesorado de otros centros
Planificación de proyectos conjuntos con profesorado de otras asignaturas, especialmente con el de instrumentos


ProspeCTIVA DE FUTURo DEL PROCESO DE ENSEÑANZA-APRENDIZAJE DEL LENGUAJE MUSICAL EN ESPAÑA
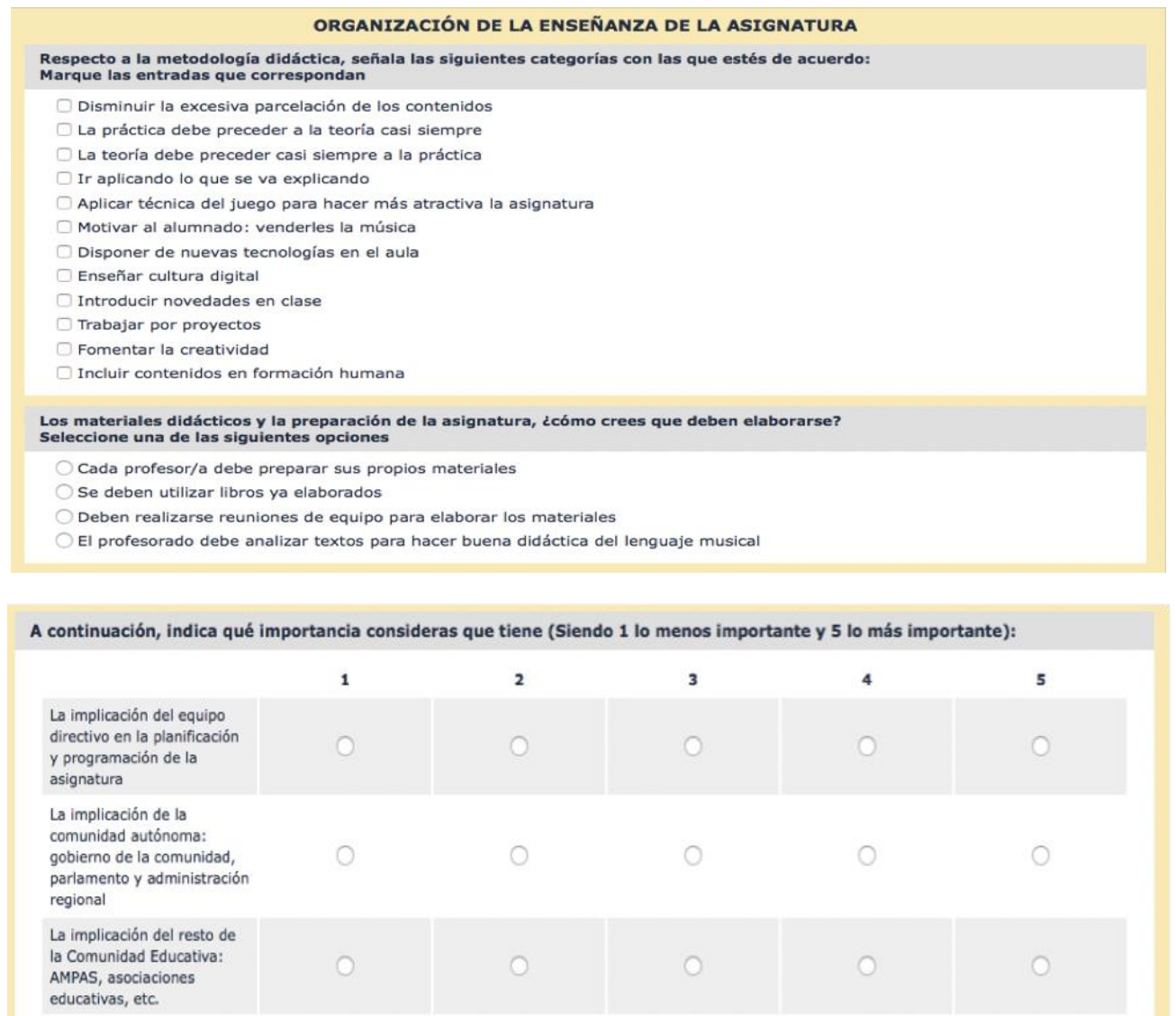

FORMACIÓN DEL PROFESORADO

En cuanto al profesorado, ¿cuál es la formación específica o cursos en los que crees que debería formarse el profesorado de Lenguaje Musical?

Marque las entradas que correspondan

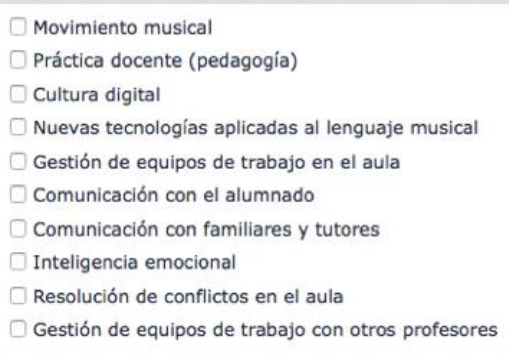

\section{OPINIÓN GENERAL}

¿Cómo describirías la situación de la música en el sistema educativo en general? Marque las entradas que correspondan

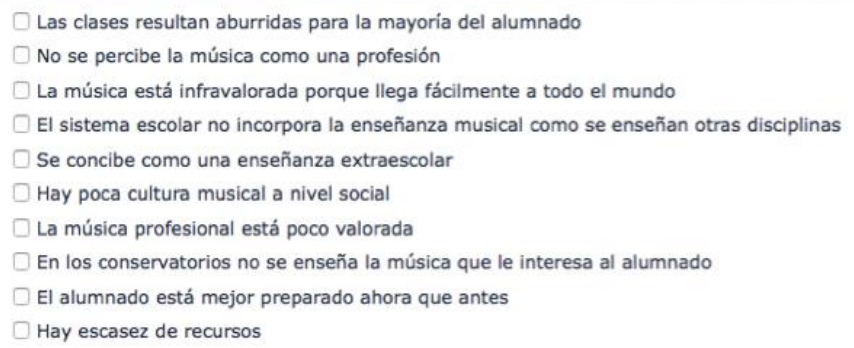


MARÍA JOSÉ SÁNCHEZ PARRA

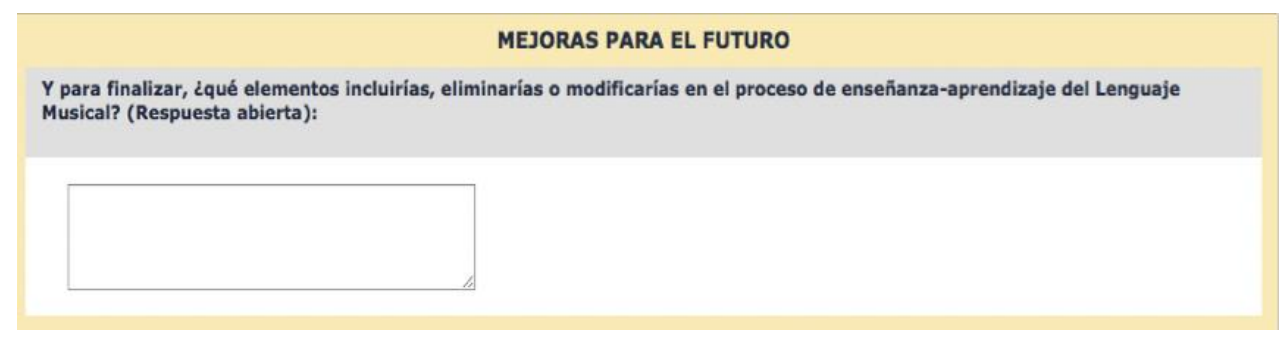

\section{ANÁLISIS DE RESUlTADOS}

Como ya hemos explicado en la metodología de investigación, la triangulación parte del cruce de los análisis tanto de las entrevistas en profundidad, como del Focus Group y la encuesta, por lo tanto, a continuación, vamos a ir detallando cada uno de estos aspectos.

En cuanto a las teorías previas las tendencias que nos encontramos son:

- Se impone la práctica musical a la teoría musical.

- La educación auditiva gana importancia.

- Se imponen las TIC como herramienta fundamental en la enseñanza del LM.

- Mejoras en las leyes educativas.

- Mayor regularización y profesionalización de las enseñanzas artísticas.

- Pequeños cambios en las programaciones didácticas.

Los ítems extraídos de las entrevistas en profundidad y de los Focus Groups fueron los siguientes:

- Visión general de la experiencia de enseñanza-aprendizaje del LM.

- Evolución de los planes de estudio.

- Formación del profesorado.

- Coordinación pedagógica.

- Organización escolar.

- Actividad didáctica.

- Percepción externa del Lenguaje Musical.

Una vez extraídos los ítems de las entrevistas en profundidad y de los Focus Group, se elaboró un cuestionario para ser lanzado de forma masiva a todos los profesores expertos 


\section{Prospectiva de FUtURo Del PROCESO DE ENSEÑANZA-APRENDIZAJE DEL LENGUAJE MUSICAL EN ESPAÑa}

de Lenguaje Musical que imparten esta materia en los Conservatorios Profesionales de Música de España.

Dado que, a través de las estadísticas oficiales del Ministerio de Educación, Cultura y Deporte del curso 2015/2016, sólo se disponía del número de docentes que imparten docencia en los Conservatorios Profesionales en todas las especialidades, y que, por lo tanto, no se disponía de un censo oficial con la referencia concreta de los profesores que impartían Lenguaje Musical, fue determinar la población de estudio mediante un censo calculado hipotético a partir de la información obtenida de las web de los Conservatorios Profesionales de Música de España.

El total de la población es: 337 profesores de Lenguaje Musical.

La falta de información homogénea tanto en el caso de las webs de las Comunidades Autónomas como en la de los propios conservatorios hizo necesario elaborar este censo hipotético aproximado en el que se realizó una estimación de 2,5 profesores de Lenguaje Musical por Conservatorio profesional de Música. Habida cuenta que hay 131 Conservatorios Profesionales de Música de España, según datos del MECD en su página web acerca del Registro Estatal de Centros Docentes No Universitarios.

El procedimiento de trabajo concreto para elaborar el censo fue:

1. Acceso y rastreo de las páginas webs de los Conservatorios para localizar los datos de referencia y de contacto de los profesores de Lenguaje Musical.

2. En aquellos casos en los que se localizaba esta información, se anotaba en una hoja de cálculo las siguientes columnas: Nombre y apellidos, Centro de procedencia, Correo electrónico de contacto.

3. En los casos en los que no se localizaba parte o toda la información, se guardaba provisionalmente la información del director y/o jefe de estudios y, en una segunda fase, con la información obtenida de estos, se procedía a completar la información establecida en el paso 2.

4. Tamaño muestral: 149 ( $\boldsymbol{n}$ 149) con afijación simple no ponderada.

$$
\mathrm{n}=\frac{Z^{2} \cdot p \cdot(1-p)}{\mathrm{e}^{2}}
$$

5. Error muestral. Para un nivel de confianza del 95\% (dos sigmas), y $\mathrm{P}=\mathrm{Q}$, el error máximo posible de $\pm 6 \%$ para el conjunto de la muestra y en el supuesto de muestreo aleatorio simple.

6. Procedimiento muestreo: Muestreo aleatorio a partir del directorio de correos electrónicos elaborado previamente en el desarrollo del censo, sin cuotas de sexo, edad o comunidad autónoma de residencia.

Al cuestionario respondieron 155 profesores expertos de las diferentes Comunidades 


\section{MARÍA JosÉ SÁNCHEZ PARRA}

Autónomas de España. De las 17 Comunidades Autónomas que forman España, no hemos obtenido ninguna respuesta de Asturias ni de las Islas Baleares, y de las dos Ciudades Autónomas tan sólo Ceuta ha participado en la encuesta.

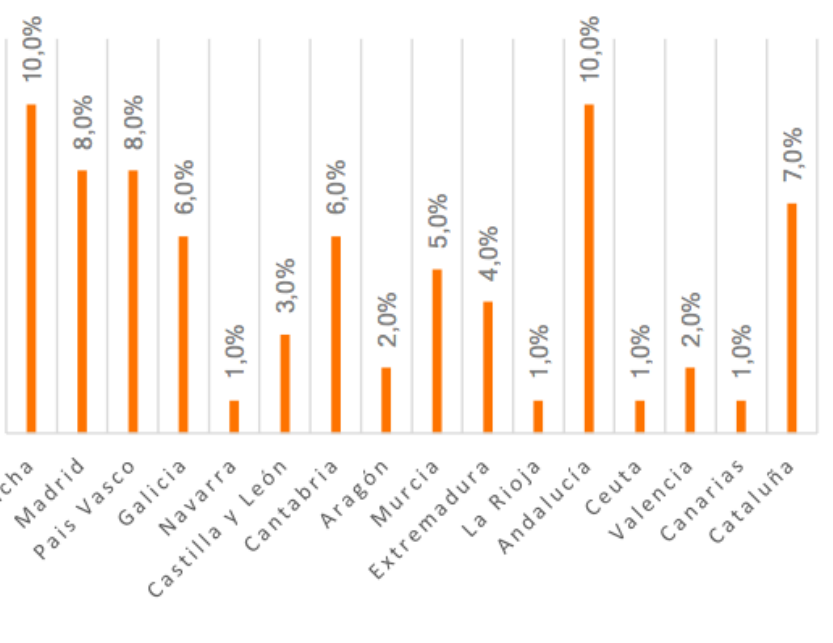

Gráfico 1: Comunidades Autónomas participantes en la Encuesta. Fuente: Elaboración Propia.

El 34\% de los docentes que contestaron a la encuesta se encuentran en un rango de edad de entre 46 y 55 años, un 32\% entre 36 y 45 años, un 19\% tienen entre 26 y 35 años, mientras que un 15\% entre 56 y 65 años. Además, cabe destacar que el $72 \%$ de los docentes que contestaron fueron mujeres, frente al $28 \%$ que fueron hombres.
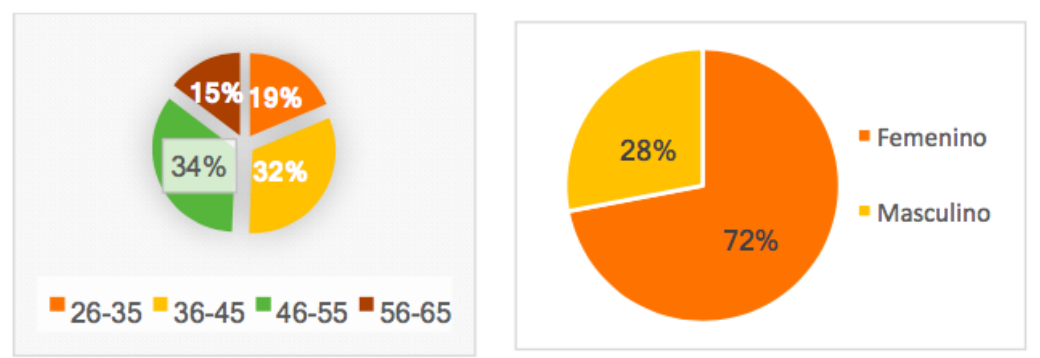

Gráfico 2: Descripción de la muestra.

Fuente: Elaboración Propia.

Con respecto al futuro de la asignatura, se hicieron preguntas relacionadas con posibles mejoras para la metodología didáctica, la secuenciación e importancia de las dimensiones que engloban el Lenguaje Musical y el contenido de dicha materia. 


\section{Prospectiva de Futuro Del PRoCeso de ENSEÑanZa-APRENDizaje DEL LENGUAJE MUSICAL EN ESPAÑA}

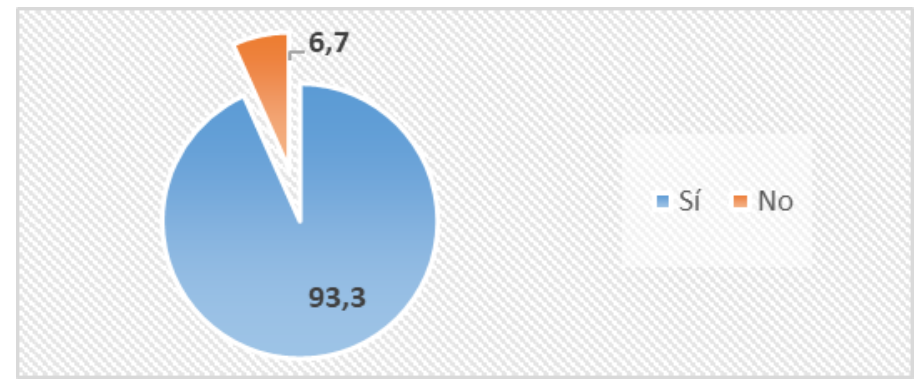

Gráfico 3: ¿Debería sufrir cambios la metodología didáctica actual? Fuente: Elaboración propia.

El 93,3\% de los actuales profesores de Lenguaje Musical, opinan que la metodología didáctica debería renovarse en un futuro, mientras que el 6,7\% opinan que no debería cambiar.

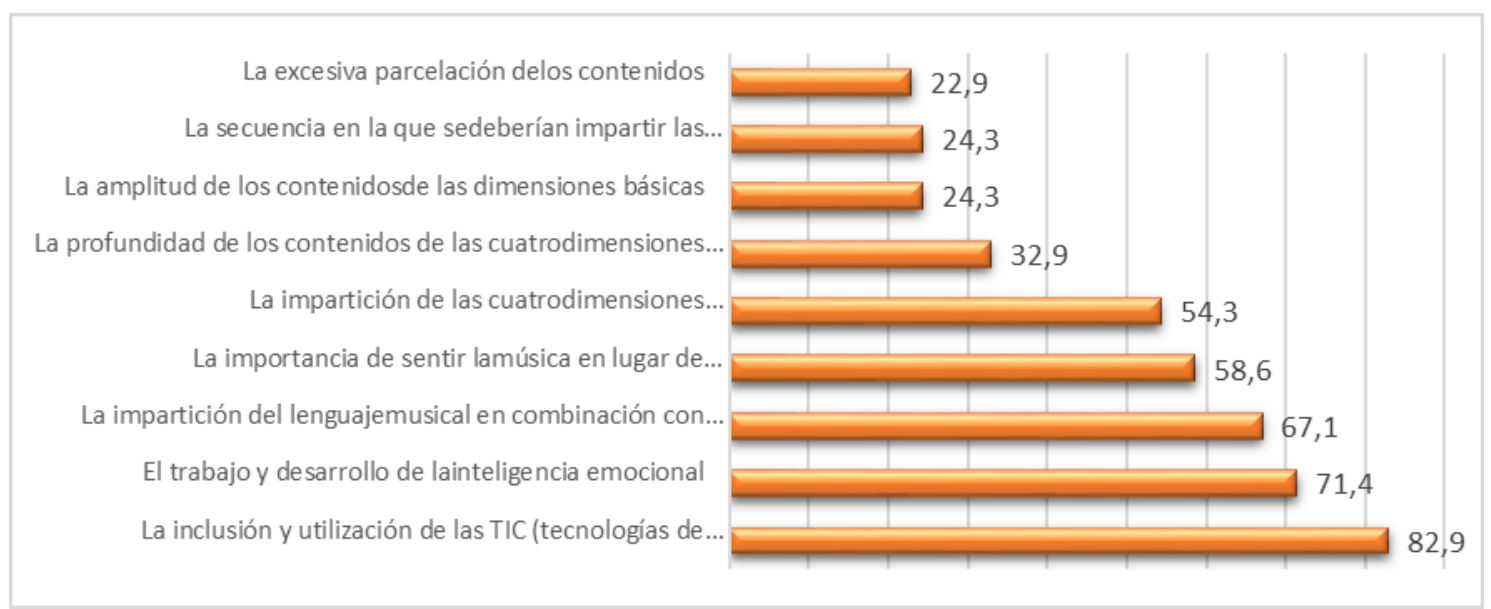

Gráfico 4. Cambios metodológicos para el futuro.

Fuente: Elaboración propia.

Los cambios que demandan los docentes para el futuro son el 82,9\% la inclusión y utilización de las TIC, el 71,4\% creen que habría que trabajar y desarrollar la inteligencia emocional, el $67,1 \%$ la impartición del Lenguaje Musical en combinación con el instrumental, el 58,6\% la importancia de sentir la música en lugar de valorar únicamente la memoria, el 54,3\% la importancia de las cuatro dimensiones básicas combinadamente, el $32,9 \%$ la profundidad de los contenidos de las cuatro dimensiones básicas, el 24,3\% la amplitud de los contenidos de las dimensiones básicas y la secuencia en la que se deberían impartir las cuatro dimensiones básicas y el 22,9\% la excesiva parcelación de los contenidos. 


\section{MARÍA JOSÉ SÁNCHEZ PARRA}

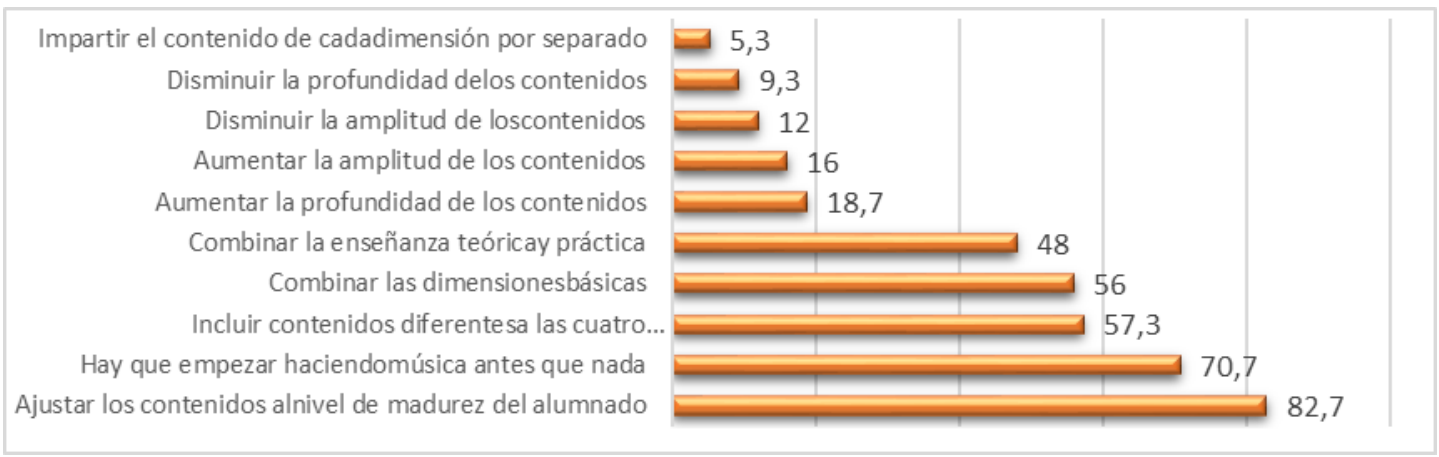

Gráfico 5. Contenidos del Lenguaje Musical.

Fuente: Elaboración propia.

En el Gráfico 5, referente a los contenidos de la materia de Lenguaje Musical, observamos cómo el 82,7\% opina que habría que ajustar los contenidos al nivel de madurez del alumnado, el 70,7\% que hay que empezar haciendo música antes que nada, el $57,3 \%$ cree que hay que incluir contenidos diferentes a las cuatro dimensiones básicas, el $56 \%$ piensa que hay que combinar las dimensiones básicas, el $48 \%$ que hay que combinar la enseñanza teórica y práctica, el 18,7\% que hay que aumentar la profundidad de los contenidos, el 16\% que hay que aumentar la amplitud de los contenidos, el $12 \%$ que hay que disminuir la amplitud de los contenidos. El 9,3\% que hay que disminuir la profundidad de los contenidos y el 5,3\% que hay que impartir el contenido de cada dimensión por separado.

\section{Discusión}

Las metodologías utilizadas en el acto didáctico del Lenguaje Musical, no están acordes a las necesidades actuales de los estudiantes.

\section{FOCUS GROUP:}

(FAM) 4: sigo trabajando por parcelas. Quizá eso tengo que mejorarlo, o pensarlo cómo poder hacerlo diferente. Siempre hay muchas cosas que cambian, pues para mejorar el aprendizaje del alumno.

(LPdeL)5: Pero hay una cosa que me encantaría poder hacer, y es lograr, por lo menos en algún curso, hacer un trabajo por proyectos. Es decir, no pretender que la clase sea autocontenida, sino que vamos a buscar una meta para lograr en cuatro clases. Una creación colectiva, un trabajo durante cuatro clases sobre una obra musical. Y eso es algo que me da miedo porque me da miedo el perder el ritmo de todo lo demás.

\footnotetext{
${ }^{4}$ Francisco Asís Arillo Mínguez, es Titulado Superior de Música en Pedagogía del Lenguaje y la Educación Musical y Dirección en el Real Conservatorio Superior de Música de Madrid. Realiza el Máster en Creación e Interpretación Musical en la Universidad Rey Juan Carlos. Actualmente es profesor de Lenguaje Musical en el Conservatorio Profesional de Música de Amaniel y en el de Danza Carmen Amaya.

${ }^{5}$ Luis Ponce de León Barranco. Doctor en Ciencias de la Educación, Profesor superior de Piano y Solfeo, BA (Hons) en Humanidades e Ingeniero de Telecomunicación. Ha ejercido como docente en el Real Conservatorio Superior de Música de Madrid, Universidad Complutense y Conservatorio Arturo Soria, labor que compagina con la composición, interpretación e investigación.
} 


\section{ProspeCtIVA DE FUTURo DEL PROCESO DE ENSEÑANZA-APRENDIZAJE DEL LENGUAJE MUSICAL EN ESPAÑA}

Pero es uno de los objetivos que tengo. Y otra cosa que se me ha escapado, intentar relacionar más todavia con el repertorio. En enseñanzas profesionales ya más o menos encuentro cosas que se adecuan a los contenidos, pero intentar que haya más música, más cultura musical, aunque los dictados sean también bellas melodias, por supuesto, y las lecciones sean bellas melodias. Oye, todo tiene que ser hermoso en la clase. Pero que se vayan impregnando también de la música de los grandes compositores, esa es una de mis metas.

$(\mathrm{MB})^{6}$ : cómo puedo hacer que mis alumnos, sin estudiar, sean capaces de ser grandes músicos. Ese es el mayor reto de mi vida.

(JFC) ${ }^{7}$ : la enseñanza debería ser un poco más humana, y no tan histérica.

¿Las nuevas tecnologías tienen cabida dentro de la enseñanza del Lenguaje Musical?

Todos los miembros del Focus Group de Toledo contestaron: "Por supuesto".

\section{ENCUESTA:}

1. Hay que renovar las metodologías: $93.3 \%$

2. Los cambios que demandan los docentes para el futuro son el $82,9 \%$ la inclusión y utilización de las TIC, el 71,4\% creen que habría que trabajar y desarrollar la inteligencia emocional, el 67,1\% la impartición del Lenguaje Musical en combinación con el instrumental, el 58,6\% la importancia de sentir la música en lugar de valorar únicamente la memoria, el 54,3\% la importancia de las cuatro dimensiones básicas combinadamente, el $32,9 \%$ la profundidad de los contenidos de las cuatro dimensiones básicas, el 24,3\% la amplitud de los contenidos de las dimensiones básicas y la secuencia en la que se deberían impartir las cuatro dimensiones básicas y el 22,9\% la excesiva parcelación de los contenidos.

Los datos verifican que: "Las metodologías utilizadas en el acto didáctico del Lenguaje Musical, no están acordes a las necesidades actuales de los estudiantes".

Los docentes de Lenguaje Musical necesitan cierta formación complementaria,

\footnotetext{
${ }^{6}$ Mónica Balo González es Doctora en Innovación e Investigación en Didáctica por la UNED y Máster en Creación e Interpretación Musical por la URJC. Titulada Superior de Solfeo, Piano, Música de Cámara y Pedagogía Musical. Diplomada en Composición y Violoncelo. Ha sido Asesora Técnico-Docente de Música y Artes Escénicas en la Consejería de Educación de la Xunta de Galicia y en el Ministerio de Educación de Madrid. En la actualidad, es profesora titular de Lenguaje Musical, Piano Complementario y Pedagogía en el Conservatorio Profesional de Música de Getafe.

${ }^{7}$ José Félix Cabello es titulado superior en Piano, Música de Cámara y Lenguaje Musical. Formación en Orff (diversos cursos de pedagogía en torno al movimiento, la danza tradicional, el canto, percusión corporal... además de completar los 3 Levels por la San Francisco School de California). En la actualidad profesor de Lenguaje Musical en el Conservatorio Profesional de Música "Teresa Berganza" de Madrid.
} 


\section{MARÍA JosÉ SÁNCHEZ PARRA}

especialmente en nuevas tecnologías e inteligencia emocional.

\section{FOCUS GROUP:}

(LPdeL): Yo creo que al profesor de Lenguaje Musical cualquier cosa le va a venir bien para su clase, eso lo primero. Cualquier conocimiento de metodologias, de repertorio, de aspectos relacionados con el movimiento, con la percusión... y quizás añadir también el tema de las nuevas tecnologias, que es algo también fundamental.

(MB): Necesitamos esa formación ya, porque para el alumnado es muy bueno.

(JFC): la enseñanza debería ser un poco más bumana, y no tan bistérica.

(LPdeL): Todos los aspectos emocionales son imprescindibles en la clase.

\section{ENCUESTA:}

1. Los resultados muestras que el $82,7 \%$ formación en nuevas tecnologías aplicadas al Lenguaje Musical, el 72\% formación en inteligencia emocional, el 57,3\% coinciden en formación tanto gestión de equipos de trabajo con otros profesores como en comunicación con el alumnado, el 53,3\% formación en movimiento musical, el 45,3\% coinciden tanto en formación para una mejora en la comunicación con familiares y tutores así como en gestión de equipos de trabajo en el aula, el $42,7 \%$ en resolución de conflictos en el aula y el $40 \%$ piensan que deberían tener formación en cultura digital.

Los datos verifican que: "Los docentes de Lenguaje Musical necesitan cierta formación complementaria, especialmente en nuevas tecnologias e inteligencia emocional”.

\section{CONCLUSIONES}

Aunque a lo largo de la historia el proceso de enseñanza- aprendizaje ha sufrido numerosos cambios, la mayoría de los expertos en la materia creen que la metodología didáctica debería cambiar en un futuro de cara al alumnado actual al que nos enfrentamos hoy en las aulas, apostando por una mayor inclusión de las TIC, el trabajo y desarrollo de la inteligencia emocional, el trabajo combinado de las dimensiones que engloban la enseñanza del Lenguaje Musical abandonando la parcelación de cada una de ellas y fomentar la creatividad.

Para poder cambiar la metodología, la formación del profesorado es muy importante, y por eso, los propios expertos proponen más prácticas docentes, formación en tecnologías aplicadas al Lenguaje Musical, formación en inteligencia emocional, formación en movimiento musical y formación en gestión de equipos de trabajo.

En cuanto a los contenidos del Lenguaje Musical, se debería ajustar al nivel de madurez del alumnado, y se debería incluir contenidos diferentes a las cuatro dimensiones del Lenguaje Musical, sobre esto último, ya hay Comunidades Autónomas que lo contemplan en sus Decretos, pero aún son muchas CCAA, las que basan sus leyes exclusivamente en los cuatro bloques de Ritmo, Audición, Entonación y Teoría.

Las metodologias del Lenguaje Musical necesitan un cambio y por tanto una formación específica y 


\section{Prospectiva de FUtURo Del PROCESO DE ENSEÑANZA-APRENDIZAJE DEL LENGUAJE MUSICAL EN ESPAÑa}

complementaria para el profesorado. Para el 96,3\% de los encuestados, las metodologías didácticas del Lenguaje Musical necesitan un cambio de cara al futuro. De las conclusiones extraídas de los expertos entrevistados, sabemos que los cambios en las leyes educativas fueron más estructurales que formales, ya que la administración no ponía al servicio del profesorado los medios adecuados para llevar a cabo dichos planes. iscepepiPor tanto, y según lo que reclaman los profesores en los Focus Group y en la encuesta, los docentes de Lenguaje Musical necesitan formación en Pedagogía, en Inteligencia emocional, en gestión de grupos y en manejo de recursos tecnológicos.

Las TIC se han convertido en herramientas indispensables dentro del proceso educativo, y se imponen como recurso fundamental para ciertos aprendizajes del Lenguaje Musical de cara al alumnado actual. El 77,3\% del profesorado de Lenguaje Musical, está de acuerdo con disponer de nuevas tecnologías en el aula. El estudio de Luis Ponce de León y Pilar Lago (2015) sobre la excursión virtual, por ejemplo, nos permite recibir clases magistrales o visitar salas de concierto sin necesidad de salir de nuestra clase.

\section{BIBLIOGRAFÍA}

LIBROS

Abrams, M.H. (1971). The Mirror and the Lamp: Romantic Theory and the Critical Tradition. London: Oxford University Press.

Berrón, E. (2016). Iniciación a la educación auditiva desde un contexto tonal en la asignatura de Lenguaje Musical (Tesis Doctoral). Universidad de Valladolid.

Boecio, A. M. T. S. (2005). Tratado de Música (Salvador Villegas Guillén, trad.). Madrid: Editorial Clásicas Ediciones. (Obra original publicada ca. 500).

Campbell, P. S. (1998). Songs in Their Heads. Music and its Meaning in Children's Lives. New York: Oxford University Press.

Casiodoro, M. A. (1865). Variarum Librim duodecim, en Casiodoro, M. A.: Opera omnia, v. 1 [Patrologia latina 69], J.-P. París: Migne editor, pp. 501-880. Recuperado de: http://patristica.net/latina/\#t069

Díaz, B. M. y Riaño, M.E. (2007). Creatividad en educación musical. Santander: Universidad de Cantabria. 


\section{MARÍA JosÉ SÁNCHEZ PARRA}

Guido d'Arezzo. (1991). Micrologus (Maurel Carter, Luminiata Florea, and Tomas J. Mathiesen, versión electrónica.). Thesaurus musicarum latinarum (Obra original publicada ca.1026). Recuperado de: http://www.chmtl.indiana.edu/tml/9th11th/GUIMIC_TEXT.html

Guido d'Arezzo. (2015). Carta de Guido dirigida al monje Miguel acerca de un canto desconocido /

Guido de Arezzo. (Carlos Rafael Domínguez, trad.) Mar del Plata: Universidad Nacional de Mar del Plata, GIEM (Obra original publicada ca. 1028). Recuperado de: https://goo.gl/6WkgC5

López de Arenosa, E. (2004). Apuntes sobre didáctica musical. Madrid: Editorial Enclave Creativa Ediciones S.L.

Misas, M.C. y Tobón, A. (2007). Dicta que dicta. Medellín: Editorial Universidad de Antioquía.

Perandones, M.A. (2010). 10 temas para la Oposición de Lenguaje Musical al cuerpo de profesores de Música y Artes Escénicas. Madrid: Ediciones Toys And Dreams Music.

San Isidoro de Sevilla (2004). Etimologías de San Isidoro de Sevilla (José Oroz y Manuel A. Marcos, trads.). Madrid: Biblioteca de autores cristianos (Obra original publicada ca. 627-630).

Saussois, N., Dutilleul, M., y Gilabert, H. (1992). Los niños de 4 a 6 años en la escuela infantil. Madrid: Ediciones Narcea S.A.

Willems, E. (2001). El oído musical. Barcelona: Ediciones Paidós Ibérica, S.A..

Willems, E. (2011). Las bases psicológicas de la educación musical. Barcelona: Ediciones Paidós Ibérica, S.A.

\section{ARTÍ́CUlos}

Balo, M., Lago, P y Ponce de León, L. (2014). "Los alumnos ante el dictado musical. Las TIC como aliadas para mejorar las experiencias". Revista científica de opinión y divulgación 28, pp. 1-14. Recuperado de: https://ddd.uab.cat/pub/dim/dim_a2014m3n28/dim_a2014m3n28a3.pdf

Barceló, B.J. (1995). "Las funciones del canto". Música y Educación: Revista trimestral de pedagogía musical, 24, pp. 37-48. 


\section{Prospectiva de FUtURo Del PROCESO DE ENSEÑANZA-APRENDIZAJE DEL LENGUAJE MUSICAL EN ESPAÑA}

Bernabé, M.M. (2015). "El lenguaje musical y las nuevas tecnologías en las enseñanzas profesionales de Música”. Revista Internacional de Tecnologías en la Educación 2, (2), pp. $79-87$.

Cabero, J. (2007). "Las necesidades de las TIC en el ámbito educativo: oportunidades, riesgos y necesidades". Tecnología y Comunicación Educativas, 45, pp. 6-19.

Dalcroze, J. (1898). Les études musicales et l'éducation del'oreille. En: Le Rythme, la Musique et l’Education. Lausanne: Foetish frères S.A.editeurs, pp. 9-12.

Gértrudix, F. y Gértrudix, M. (2007). "Investigaciones en torno a las TIC en educación: una panorámica actualizada". Docencia e Investigación, 17, pp. 119-146.

Hernández, J. R., Hernández, J. A., y De Moya, M. V. (2011). “Las bandas sonoras como base de la audición activa: experiencias educativas para el desarrollo musical infantil". Ensayos. Revista de la Facultad de Educación de Albacete, 26, pp. 165-178

Laucirica, A., Ordoñana, J. A. y Muruamendiaraz, N. (2009). “Consideraciones preliminares en el diseño de programas informáticos para el desarrollo rítmico". Revista Electrónica de LEEME, 24, pp. 49-63.

Marchesi, A. (2012). "Preámbulo”. En R. Carneiro, J.C. Toscano y T. Díaz (coords.), Los desafios de las TIC para el cambio educativo, pp. 7-9. Madrid: OEI.

Molina, E. (1998). "La improvisación y el lenguaje musical". Eufonía: Didáctica de la música, 11, pp. 59-76.

Ponce de León, L. y Lago, P. (2015). "La excursión virtual como estrategia didáctica en el aula de música y de otras materias. Fortalezas y limitaciones”. Revista DIM, 11 (32), pp. 1-16 Recuperado de: https://goo.gl/fjNeJN

Sustaeta. I, y Domínguez-Alcahud, M.P. (2004). “Aplicaciones didácticas de la informática musical". Revista Electrónica Complutense de Investigación en Educación Musical 1 (4), pp. 1 12. Recuperado de: http://pendientedemigracion.ucm.es/info/reciem/v1n4.pdf

Torres Gallardo, B. (2013). "La voz y nuestro cuerpo. Un análisis funcional”. Revista de Investigaciones en Técnica Vocal 1 (1), pp. 40-58. Recuperado de: https://goo.gl/LvAG86 
MARÍA JOSÉ SÁNCHEZ PARRA

Valencia, R., y Ventura, E. (2003). "El abandono de los estudios musicales de grado elemental en el Conservatorio Superior de Música de Las Palmas de Gran Canaria”. Anuario de filosofía, psicología y sociología, 6, pp. 77-100.

Fecha de recepción: 30/03/2018

Fecha de aceptación: 05/10/2018 